\title{
Sinc-Fractional Operator on Shannon Wavelet Space
}

\author{
Carlo Cattani* \\ Engineering School, DEIM, Tuscia University, Viterbo, Italy
}

In this paper the sinc-fractional derivative is extended to the Hilbert space based on Shannon wavelets. Some new fractional operators based on wavelets are defined. One of the main task is to investigate the localization and compression properties of wavelets when dealing with the non-integer order of a differential operator.

Keywords: fractional calculus, shannon wavelet, sinc-function, operational matrix, connection coefficients

\section{OPEN ACCESS}

Edited by:

Dumitru Baleanu,

University of Craiova, Romania

Reviewed by:

Xiao-Jun Yang,

China University of Mining and

Technology, China

Jordan Yankov Hristov,

University of Chemical Technology

and Metallurgy, Bulgaria

Abdullahi Yusuf,

Federal University, Nigeria

*Correspondence:

Carlo Cattani

cattani@unitus.it

Specialty section:

This article was submitted to

Mathematical Physics,

a section of the journal

Frontiers in Physics

Received: 30 August 2018 Accepted: 28 September 2018

Published: 24 October 2018

Citation:

Cattani C (2018) Sinc-Fractional Operator on Shannon Wavelet Space.

Front. Phys. 6:118.

doi: 10.3389/fphy.2018.00118

\section{INTRODUCTION}

In recent years, fractional calculus has been growing fast both in theory and applications to many different fields. Several classical and fundamental problems have been revised by using fractional methods, thus showing unexpected new results [1-3], while more and more new problems were shaped to fit the theoretical models of fractional calculus [4-7].

In fractional calculus is based on two universally accepted principles: the first one is that the definition of fractional derivative is not unique, thus giving raise to a neverending controversial debate on the best fractional operator. The second principle is that, although the missing uniqueness of the fractional operator, fractional calculus is an essential tool for a deeper and more comprehensive investigation of complex, non-linear, local, or non-local problems.

Therefore according to the suitable choice of the fractional differential operator, there follows a corresponding model of analysis so that the physical model and the corresponding physical interpretation of the results it strongly depends on the chosen fractional operator.

In some recent papers [8-15] the classical Lie symmetry analysis has been combined with the Riemman-Liouville fractional derivative to solve time fractional partial differential equations. In these papers, Lie point symmetries have been used to convert a fractional partial differential equation into a non-linear ordinary differential equation, that can be solved by suitable methods. Some fractional operators have been used also to study non-differentiable functions [see e.g., [16] some of them are more suitable for the analysis of non-differentiable sets, or fractal sets like the Cantor fractal set [4-7] Some fractional operators have been specially defined to analyze complex functions [17-19]. For instance the chaotic decay to zero of the complex $\zeta$-Riemann function was easily shown by using a suitable fractional derivative [19].

Among the many interesting definitions of fractional operators, some Authors have recenlty proposed a fractional differential operator based on the sinc-function [20]. This function is very popular in the signal analysis, also because it is a localized function with slow decay. Moreover, it is the fundamental basic function for the definition of the so-called Shannon wavelet theory, i.e., the multiscale analysis on Shannon wavelets [21-26].

This paper will focus on the definition of a fractional derivative by the Shannon wavelets. These functions belong to a special family of wavelets which have a sharp compact support in the 
frequency space, so that their Fourier transform are boxfunctions in frequencies. This is a great advantage because, the frequency domain of a signal can be easily decomposed in terms of scaled box-functions.

Wavelet theory has been growing very fast so that there has been also a wide spreading of wavelets for the solution of theoretical and applied problems. However, alike the various definition of fractional operators there exist also many different families of wavelets and this missing uniqueness it might be considered as a drawback because of the arbitrary choice. Nevertheless all families of wavelets enjoy two fundamental properties their localization in time (or frequency) and the multiscale decomposition. Due to their localization they can be used to detect, and single out, localized singularities and/or peaks, while the multiscale property enable to decompose the approximation space into separate scales [27]. Thanks to these properties wavelets have been used to solve non-linear problems and moreover they are the most suitable tool for the analysis of multiscale problems.

The sinc-fractional operator will be generalized in order to compute the fractional derivative of the $L_{2}(\mathbb{R})$-functions belonging to the Hilbert space defined by the Shannon wavelet. In doing so, we will be able to compute the fractional derivative of these functions by knowing only their wavelet coefficients. Moreover, with this approach we will be able to decompose the fractional derivative at different scales, thus showing the influence of a given scale in multiscale physical problems.

The organization of this paper is as follows: Preliminary remarks on fractional operators are given in section 2 . In section 3 the sinc fractional derivative, as given by Yang et al. [20] is described. Section 4 gives the basic properties on the multiscale approximation defined on Shannon wavelet. The differential properties of the functions belonging to the Hilbert space based on Shannon wavelet are given in section 5, together with the explicit form of the integer order derivatives (see also $[24,26]$ ). Section 6 deals with the sinc-fractional derivative on the Hilbert space based on Shannon wavelets, i.e., sinc-fractional derivative of functions which can be represented as Shannon wavelet series.

\section{PRELIMINARY REMARKS}

In this section some of the most popular definition of fractional derivatives [28-30] are given.

Let us start with the Riemann-Liouville derivative.

Definition 1. The Riemann-Liouville integral of fractional order $v \geq 0$ of a function $f(x)$, is defined as

$$
\left(J^{v} f\right)(t)=\left\{\begin{array}{l}
\frac{1}{\Gamma(v)} \int_{0}^{t}(t-\tau)^{\nu-1} f(\tau) d \tau, v>0 \\
f(t), \quad v=0
\end{array}\right.
$$

The Riemann-Liouville fractional operator $J^{\alpha}$ has the following properties:

(a) $J^{\alpha}\left(J^{\beta} f(t)\right)=J^{\beta}\left(J^{\alpha} f(t)\right)$,

(b) $J^{\alpha}\left(J^{\beta} f(t)\right)=J^{\alpha+\beta} f(t)$,

(c) $J^{\alpha} t^{v} \quad=\frac{\Gamma(v+1)}{\Gamma(\alpha+v+1)} t^{v+\alpha}, \quad \alpha, \beta \geq 0, v>-1$

(d) $J^{v} e^{\lambda t} \quad=\frac{1}{v \Gamma(v)} e^{\lambda t} t^{\nu}, v>0$,

(e) $J^{v} c \quad=\frac{c}{v \Gamma(v)} t^{v}, v>0$.

From this definition there follows the corresponding derivative according to the following:

Definition 2. Riemann-Liouville fractional derivative of order $\alpha>0$ is defined as

$$
D_{R L}^{\alpha} f(t)=\frac{d^{n}}{d t^{n}} J^{n-\alpha} f(t), \quad n \in \mathbb{N}, \quad n-1<\alpha \leq n .
$$

The main problem with this derivative is the unvanishing value for a constant function, therefore it was proposed by Caputo the following $[28,29]$. then

Let $f(x) \in \mathcal{C}^{n}$ be a $n$-differentiable function, $\alpha$ a positive value,

Definition 3. The $\alpha$-order Caputo fractional derivative is defined as

$D_{C}^{\alpha} f(x)= \begin{cases}\frac{d^{n} f(x)}{d x^{n}}, & 0<\alpha \in \mathbb{N}, \\ \frac{1}{\Gamma(n-\alpha)} \int_{0}^{x} \frac{f^{(n)}(\tau)}{(x-\tau)^{\alpha-n+1}} d \tau, t>0, & 0 \leq n-1 \\ & <\alpha<n .\end{cases}$

where $n$ is an integer, $x>0$, and $f \in \mathcal{C}^{n}$.

It can be easily shown that:

(a) $J^{\alpha} D_{C}^{\alpha} f(x)=f(x)-\sum_{k=0}^{n-1} f^{(k)}\left(0^{+}\right) \frac{x^{k}}{k !}, \quad t>0$.

(b) $D_{C}^{\alpha} J^{\alpha} f(x)=f(x)$

(c) $D_{C}^{\alpha} t^{n}= \begin{cases}0, & \text { for } n \in \mathbb{N}_{0} \text { and } \alpha<n, \\ \frac{\Gamma(n+1)}{\Gamma(n-\alpha+1)} t^{n-\alpha}, & \text { otherwise. }\end{cases}$

(d) $D_{C}^{\alpha} D_{C}^{\beta} f(x)=D_{C}^{\beta} D_{C}^{\alpha} f(x)$. 


\section{SINC-FRACTIONAL DERIVATIVE}

Riemann-Liouville (RL) and Caputo (C) derivatives are the most popular derivatives and have been used in many applications (see e.g., [2, 3, 16, 18, 25, 26, 29, 31-41]), nevertheless they both suffer for some unavoidable drawbacks. In particular, the RL-derivative is unvanishing when $f(x) \neq$ constant while the $\mathrm{C}$-derivative is defined on a singular kernel. Because of that, in recent years many efforts were devoted to find some more flexible non-singular derivatives. Moreover, due to the fact that the fractional derivative is not univocally defined, there have been proposed many alternative interesting new definitions.

Indeed the more general fractional derivative with a given kernel $K(x, \alpha)$, which generalizes the $\mathrm{C}$-derivative is:

$$
D^{\alpha} f(x)=\left\{\begin{array}{l}
\frac{d^{n} f(x)}{d x^{n}}, \quad 0<\alpha \in \mathbb{N}, \\
\int_{0}^{x} f^{(n)}(\tau) K(x-\tau, \alpha) d \tau, x>0, \quad 0 \leq n-1<\alpha<n .
\end{array}\right.
$$

The kernel should be defined in a such a way that at least the two conditions

$$
\lim _{\alpha \rightarrow 0} K(x-\tau, \alpha)=1, \quad \quad \lim _{\alpha \rightarrow 1} K(x-\tau, \alpha)=\delta(x-\tau)
$$

hold true, moreover, in order to be a non-singular kernel, it should be also

$$
\lim _{x \rightarrow \tau} K(x-\tau, \alpha) \neq 0, \quad \forall \alpha .
$$

Although there are several definitions of derivatives they all depend on a kernel. In particular, it can be easily seen that the C-derivative [42], the Caputo-Fabrizio (CF) derivative [34], and the Atangana-Baleanu (AB) derivative [43] are some special cases of (3.1) corresponding respectively to the kernels:

$$
\begin{aligned}
K(x-\tau, \alpha) & =\frac{1}{\Gamma(n-\alpha)}(x-\tau)^{n-\alpha-1} \\
K(x-\tau, \alpha) & =\frac{M(\alpha)}{1-\alpha} e^{-\frac{\alpha}{1-\alpha}(x-\tau)} \\
K(x-\tau, \alpha) & =\frac{B(\alpha)}{1-\alpha} E_{\alpha}\left(-\frac{\alpha}{1-\alpha}(x-\tau)\right),
\end{aligned}
$$

where the Mittag-Leffler function is taken as

$$
E_{\alpha}(x) \stackrel{\text { def }}{=} \sum_{k=0}^{\infty} \frac{x^{\alpha k}}{\Gamma(\alpha k+1)} .
$$

It can be easily shown that all kernels (3.4) fulfill (3.2) while only $(\mathrm{CF})$ and $(\mathrm{AB})$ fulfill also the condition (3.3).

\subsection{The Yang-Gao-Terneiro Machado-Baleanu Fractional Derivative [20]}

With respect to the integration variable $\tau$ all kernels (3.4) have a decay to zero, in a such way that for a bounded $f^{(n)}(x)$ the integral $(3.1)_{2}$ converges.
Among the non-singular kernels with decay to zero a fractional derivative based on a sinc-function kernel was recently defined by Yang, Gao, Terneiro Machado, and Baleanu (YGTMB) [20].

The sinc-function, defined as Yang et al.[20]

$$
\operatorname{sinc} x \stackrel{\text { def }}{=} \frac{\sin \pi x}{\pi x}
$$

owns a quite large amount of nice properties, so that it became a fundamental tools in applied science and signal analysis. In particular, it was shown (see e.g., [20]) that, for a given $x$

$$
\lim _{\alpha \rightarrow 0} \frac{1}{\alpha} \operatorname{sinc}\left(\frac{x}{\alpha}\right)=\delta(x)
$$

being $\delta(x)$ the Dirac-delta function

$$
\delta(x)= \begin{cases}0, & x \neq 0 \\ 1, & x=0\end{cases}
$$

More in general from (3.6) it is

$$
\lim _{\alpha \rightarrow 0} \frac{1}{\alpha} \operatorname{sinc}\left(\frac{x-\tau}{\alpha}\right)=\delta(x-\tau)
$$

By using the sinc-function, we have the following definition of the sinc fractional derivative [20].

Definition 4 (Yang-Gao-Tenreiro Machado-Baleanu). The YGTMB fractional derivative is defined as Yang et al. [20]

$$
D_{Y G T M B}^{\alpha} f(x) \stackrel{\text { def }}{=} \frac{\alpha P(\alpha)}{1-\alpha} \int_{a}^{x} \operatorname{sinc} \frac{\alpha(x-\tau)}{1-\alpha} f^{(n)}(x) d \tau, 0 \leq n-1
$$

We can see that also this kernel

$$
K(x-\tau, \alpha)=\frac{\alpha P(\alpha)}{1-\alpha} \operatorname{sinc} \frac{\alpha(x-\tau)}{1-\alpha}
$$

belongs to the class of kernels (3.1). It can be also shown that this kernel fulfills the conditions (3.2),(3.3) (see [20]) being

$\lim _{\alpha \rightarrow 0} \frac{\alpha P(\alpha)}{1-\alpha} \operatorname{sinc} \frac{\alpha(x-\tau)}{1-\alpha}=1, \lim _{\alpha \rightarrow 1} \frac{\alpha P(\alpha)}{1-\alpha} \operatorname{sinc} \frac{\alpha(x-\tau)}{1-\alpha}=\delta(x-\tau)$,

and the normalization constant factor $P(\alpha)$ is such that

$$
\lim _{\alpha \rightarrow 0}[K(x, \alpha) P(\alpha)]=\lim _{\alpha \rightarrow 1}[K(x, \alpha) P(\alpha)]=1
$$


In particular, there follows from (3.8)

$$
D_{Y G T M B}^{\alpha} f^{(n)}(x)= \begin{cases}f^{(n-1)}(x)-f^{(n-1)}(0), & \alpha=0 \\ f^{(n)}(x) & , \quad \alpha=1 \\ & (0 \leq n-1<\alpha<n) .\end{cases}
$$

\subsubsection{Polynomial Approximation of the Kernel}

The sinc kernel (3.9) can be written also as an infinite product as follows. Starting from the known product:

$$
\sin x=x \prod_{k=1}^{\infty}\left(1-\frac{x^{2}}{k^{2} \pi^{2}}\right)
$$

by taking into account (3.5) it is

$$
\operatorname{sinc} x=\frac{\sin (\pi x)}{\pi x}=\frac{1}{\pi x}(\pi x) \prod_{k=1}^{\infty}\left(1-\frac{(\pi x)^{2}}{k^{2} \pi^{2}}\right)
$$

so that

$$
\operatorname{sinc} x=\prod_{k=1}^{\infty}\left(1-\frac{x^{2}}{k^{2}}\right)
$$

It should be noticed that in the interval $[-1,1]$ the sinc-function can be approximated by

$$
\operatorname{sinc} x \cong \prod_{k=1}^{n}\left(1-\frac{x^{2}}{k^{2}}\right)
$$

so that if we define as the error of approximation

$$
\varepsilon(n)=\left|\operatorname{sinc} x-\prod_{k=1}^{n}\left(1-\frac{x^{2}}{k^{2}}\right)\right|
$$

we have

$\max \varepsilon(1) \leq 0.14, \max \varepsilon(2) \leq 0.08, \quad \max \varepsilon(3) \leq$ $0.055, \max \varepsilon(4) \leq 0.04$,

so that already with $n=1$ :

$$
\operatorname{sinc} x \cong\left(1-x^{2}\right)
$$

the error of approximation in $[-1,1]$ is less that $15 \%$.

It should be noticed that with this approximation the YGTMB-derivative (3.8) becomes

$$
\begin{gathered}
D_{Y G T M B^{*}}^{\alpha} f(x)=\frac{\alpha P(\alpha)}{1-\alpha} \int_{a}^{x}\left[\begin{array}{c}
\alpha^{2} \\
\leq n-1<\alpha<n
\end{array}(x-\tau)^{2}\right] f^{(n)}(x) d \tau, \\
(1-\alpha)^{2}
\end{gathered}
$$

that is

$$
\begin{aligned}
D_{\text {YGTMB }}^{\alpha} f(x)= & \frac{\alpha P(\alpha)}{1-\alpha}\left[f^{(n-1)}(x)-f^{(n-1)}(0)\right]-\frac{\alpha^{3} P(\alpha)}{(1-\alpha)^{3}} \\
& \int_{a}^{x}(x-\tau)^{2} f^{(n)}(x) d \tau, \quad 0 \leq n-1<\alpha<n
\end{aligned}
$$

By assuming as a normalization factor

$$
P(\alpha)=\frac{(1-\alpha)^{3}}{\alpha}
$$

we get

$$
\begin{aligned}
D_{\text {YGTMB* }}^{\alpha} f(x)= & (1-\alpha)^{2}\left[f^{(n-1)}(x)-f^{(n-1)}(0)\right]-\alpha^{3} \\
& \int_{a}^{x}(x-\tau)^{2} f^{(n)}(x) d \tau, \quad 0 \leq n-1<\alpha<n
\end{aligned}
$$

so that the fractional derivative can be seen as the interpolation between the function and its derivative (as shown e.g., in Cattani $[25,26])$.

\subsection{Sinc Fractional Derivative With Unbounded Domain}

Let us consider the integral of sinc function over the unbounded domain $[-\infty, \infty]$. By a direct computation it can be shown that

$$
\int_{-\infty}^{\infty} \frac{\sin \pi x}{\pi x} d x=1, \quad \int_{-1}^{1} \frac{\sin \pi x}{\pi x} d x \cong 1.17
$$

so that the sinc-function is a function mainly localized around the origin. In fact, the sinc function is known as a function with a decay to zero, therefore we can extend the definition (3.8) over the unbounded domain $\mathbb{R}$ so that we can define the sinc fractional derivative as the YGTMB fractional derivative on the unbounded domain $\mathbb{R}$, that is

Definition 5 (sinc fractional derivative). The sinc fractional derivative $D_{S}^{\alpha}$ of a function $f(x)$ is defined as

$D_{S}^{\alpha} f(x) \stackrel{\text { def }}{=} \frac{\alpha P(\alpha)}{1-\alpha} \int_{-\infty}^{\infty} \operatorname{sinc} \frac{\alpha(x-\tau)}{1-\alpha} f^{(n)}(x) d \tau, 0 \leq n-1<\alpha<n$

where the normalization factor $P(\alpha)$ is chosen to fulfill conditions (3.2), (3.3) and the kernel is

$$
K(x-\tau, \alpha)=\frac{\alpha P(\alpha)}{1-\alpha} \operatorname{sinc} \frac{\alpha(x-\tau)}{1-\alpha} .
$$

In particular, we can also assume

$$
\frac{\alpha}{1-\alpha}=2^{\beta}
$$

so that

$$
\alpha=\frac{2^{\beta}}{1-2^{\beta}}, \quad \beta=\log _{2} \frac{\alpha}{1-\alpha}
$$

and the derivative (3.10) can be written as

$$
D_{S}^{\beta} f(x) \stackrel{\text { def }}{=}-2^{\beta} \int_{-\infty}^{\infty} \operatorname{sinc}\left(2^{\beta} \tau-2^{\beta} x\right) f^{(n)}(\tau) d \tau, \quad 2^{\beta} \leq \frac{n}{n+1} .
$$




\section{SHANNON WAVELETS}

The sinc-function plays a fundamental role also in wavelet theory. In fact, the basic functions (scaling and wavelet) of the so-called Shannon wavelets (see e.g., [21-26]) can be defined by the sinc (3.5). In this section, some remarks on Shannon wavelets and connection coefficients are shortly summarized.

\subsection{Preliminary Remarks}

Shannon wavelet theory (see e.g., [21-24]) is based on the scaling function $\varphi(x)$, also known as sinc function, and the wavelet function $\psi(x)$ respectively defined as

$$
\left\{\begin{aligned}
\varphi(x) & =\sin c x \stackrel{\text { def }}{=} \frac{\sin \pi x}{\pi x}=\frac{e^{\pi i x}-e^{-\pi i x}}{2 \pi i x} . \\
\psi(x) & =\frac{\sin 2 \pi\left(x-\frac{1}{2}\right)-\sin \pi\left(x-\frac{1}{2}\right)}{\pi\left(x-\frac{1}{2}\right)} \\
& =\frac{e^{-2 i \pi x}\left(-i+e^{i \pi x}+e^{3 i \pi x}+i e^{4 i \pi x}\right)}{2 \pi\left(x-\frac{1}{2}\right)} .
\end{aligned}\right.
$$

The second function can be expressed in terms of the first, as

$$
\psi(x)=2 \varphi(2 x-1)-\varphi\left(x-\frac{1}{2}\right)
$$

The families of translated and dilated Shannon scaling functions [21-24], are

$$
\begin{aligned}
\varphi_{k}^{n}(x) & =2^{n / 2} \varphi\left(2^{n} x-k\right)=2^{n / 2} \frac{\sin \pi\left(2^{n} x-k\right)}{\pi\left(2^{n} x-k\right)} \\
& =2^{n / 2} \frac{e^{\pi i\left(2^{n} x-k\right)}-e^{-\pi i\left(2^{n} x-k\right)}}{2 \pi i\left(2^{n} x-k\right)}, \\
& =\frac{2^{n / 2}}{2 \pi i\left(2^{n} x-k\right)} \sum_{s=0}^{\infty} \frac{\pi^{s} i^{s}}{s !}\left[1-(-1)^{s}\right]\left(2^{n} x-k\right)^{s} \\
& =\frac{2^{n / 2}}{2 \pi i\left(2^{n} x-k\right)} \sum_{s=0}^{\infty} \frac{\pi^{s} i^{s}}{s !}\left(1-e^{\pi s}\right)\left(2^{n} x-k\right)^{s} \\
& =2^{n / 2-1} \sum_{s=1}^{\infty} \frac{\pi^{s-1} i^{s-1}}{s !}\left[1-(-1)^{s}\right]\left(2^{n} x-k\right)^{s-1} .
\end{aligned}
$$

By a direct computation it can be easily shown that this series can be also written as

$$
\varphi_{k}^{n}(x)=2^{n / 2} \sum_{s=0}^{\infty}(-1)^{s} \frac{\pi^{2 s}}{(2 s+1) !}\left(2^{n} x-k\right)^{2 s}
$$

that is

$$
\varphi_{k}^{n}(x)=2^{n / 2} \sum_{s=0}^{\infty}(-1)^{s} \frac{\pi^{2 s}}{(2 s+1) !} \sum_{j=0}^{2 s}\left(\begin{array}{c}
2 s \\
j
\end{array}\right)\left(2^{n} x\right)^{j}(-k)^{2 s-j}
$$

In the special case when $k=0$, from (4.4) we have

$$
\varphi_{0}^{n}(x)=2^{n / 2} \sum_{s=0}^{\infty}(-1)^{s} \frac{\pi^{2 s}}{(2 s+1) !} 2^{2 n s} x^{2 s}
$$

while for the translated instances at the zero scale $n=0$ we obtain from (4.4)

$$
\varphi_{k}(x) \stackrel{\text { def }}{=} \varphi(x-k)=\sum_{s=0}^{\infty}(-1)^{s} \frac{\pi^{2 s}}{(2 s+1) !}(x-k)^{2 s}
$$

Analogously, the translated and dilated instances of the Shannon wavelets are

$$
\begin{aligned}
\psi_{k}^{n}(x) & =2^{n / 2} \frac{\sin 2 \pi\left(2^{n} x-k-\frac{1}{2}\right)-\sin \pi\left(2^{n} x-k-\frac{1}{2}\right)}{\pi\left(2^{n} x-k-\frac{1}{2}\right)}, \\
& =\frac{2^{n / 2}}{2 \pi\left(2^{n} x-k-\frac{1}{2}\right)} \sum_{r=1}^{2} i^{1+r} e^{r \pi i\left(2^{n} x-k\right)}-i^{1-r} e^{-r \pi i\left(2^{n} x-k\right)} .
\end{aligned}
$$

or, by taking into account (4.2)

$$
\psi_{k}^{n}(x)=2 \varphi_{k}^{n+1}(x)-\varphi_{k}^{n}\left(x-\frac{1}{2}\right)
$$

and Equation (4.3), it is

$$
\begin{aligned}
\psi_{k}^{n}(x) & =2^{n / 2} \sum_{s=1}^{\infty} \frac{\pi^{s-1} i^{s-1}}{s !}\left[1-(-1)^{s}\right]\left(2^{n} x-k\right)^{s-1} \\
& -2^{n / 2-1} \sum_{s=1}^{\infty} \frac{\pi^{s-1} i^{s-1}}{s !}\left[1-(-1)^{s}\right]\left(2^{n}\left(x-\frac{1}{2}\right)-k\right)^{s-1}
\end{aligned}
$$

From (4.9), by taking into account (4.4), it is

$$
\begin{aligned}
\psi_{k}^{n}(x)= & 2^{n / 2} \sum_{s=0}^{\infty}(-1)^{s} \frac{\pi^{2 s}}{(2 s+1) !}\left\{2^{3 / 2}\left(2^{n+1} x-k\right)^{2 s}\right. \\
& \left.-\left[\left(2^{n} x-k\right)-2^{n-1}\right]^{2 s}\right\}
\end{aligned}
$$

so that at the zero scale $n=0$ it is

$$
\begin{aligned}
& \psi_{k}(x) \stackrel{\text { def }}{=} \psi_{k}^{0}(x)=\psi(x-k)=\sum_{s=0}^{\infty}(-1)^{s} \frac{\pi^{2 s}}{(2 s+1) !}\left\{2^{3 / 2}\right. \\
& \left.(2 x-k)^{2 s}-\left[(x-k)^{2 s}-\frac{1}{2}\right]^{2 s}\right\}
\end{aligned}
$$

and, at the origin $k=0$

$$
\begin{aligned}
& \psi^{n}(x) \stackrel{\text { def }}{=} \psi_{0}^{n}(x)=2^{n / 2} \sum_{s=0}^{\infty}(-1)^{s} \frac{\pi^{2 s}}{(2 s+1) !}\left\{2^{3 / 2}\left(2^{n+1} x\right)^{2 s}\right. \\
& \left.-\left(2^{n} x-2^{n-1}\right)^{2 s}\right\} .
\end{aligned}
$$


By assuming,

$$
\begin{aligned}
& \varphi_{0}^{0}(x)=\varphi(x), \psi_{0}^{0}(x)=\psi(x), \varphi_{k}^{0}(x)=\varphi_{k}(x)=\varphi(x-k) \\
& \psi_{k}^{0}(x)=\psi_{k}(x)=\psi(x-k)
\end{aligned}
$$

and taking into account $(4.4),(4.10)$ the fundamental functions $\varphi(x), \psi(x)$, can be expressed as the power series

$$
\left\{\begin{array}{l}
\varphi(x)=\sum_{s=0}^{\infty}(-1)^{s} \frac{\pi^{2 s}}{(2 s+1) !} x^{2 s} \\
\psi(x)=\sum_{s=0}^{\infty}(-1)^{s} \frac{\pi^{2 s}}{(2 s+1) !}\left[2^{2 s+3 / 2} x^{2 s}-\left(x-\frac{1}{2}\right)^{2 s}\right]^{(4.11)}
\end{array}\right.
$$

\subsection{Properties of the Shannon Wavelet}

Shannon wavelets enjoy some interesting properties. In particular, when they are evaluated at some special points they assume some very simple expressions. For instance, according to (4.3), it is

$$
\varphi_{k}(h)=\varphi_{h}(k)=\varphi(h-k)=\varphi(k-h)=\delta_{k h}, \quad(h, k \in \mathbb{Z}),
$$

so that

$$
\varphi_{k}(h)=\delta_{k h}=\left\{\begin{array}{lll}
0, & h \neq k, & (h, k \in \mathbb{Z}) \\
1, & h=k, & (h, k \in \mathbb{Z})
\end{array}\right.
$$

Analogously we have [24]

$$
\begin{gathered}
\psi_{k}^{n}(h)=(-1)^{2^{n} h-k} \frac{2^{1+n / 2}}{\left(2^{n+1} h-2 k-1\right) \pi}, \quad\left(2^{n+1} h-2 k-1 \neq 0\right) \\
\psi_{k}^{n}(x)=0, \quad x=2^{-n}\left(k+\frac{1}{2} \pm \frac{1}{3}\right), \quad(n \in \mathbb{N}, k \in \mathbb{Z}) \\
\lim _{x \rightarrow 2^{-n}\left(h+\frac{1}{2}\right)} \psi_{k}^{n}(x)=-2^{n / 2} \delta_{h k},
\end{gathered}
$$

being,

$$
\psi_{k}^{0}(0)=(-1)^{k+1} \frac{2}{(2 k+1) \pi}
$$

and since $k \in \mathbb{Z}, 2 k+1 \neq 0$.

It can be shown (see e.g., [25]) that both scaling and wavelet functions are bounded, being:

$$
\begin{gathered}
\max \left[\varphi_{k}\left(x_{M}\right)\right]=1, \quad x_{M}=k, \\
\max \left[\psi_{k}^{n}\left(x_{M}\right)\right]=2^{n / 2} \frac{3 \sqrt{3}}{\pi}, \quad x_{M}=\left\{\begin{array}{l}
-2^{-n}\left(k+\frac{1}{6}\right) \\
\frac{2^{-n-1}}{3}(18 k+7),
\end{array}\right.
\end{gathered}
$$

and

$$
\lim _{x \rightarrow \pm \infty} \varphi_{k}^{n}(x)=0, \quad \lim _{x \rightarrow \pm \infty} \psi_{k}^{n}(x)=0 .
$$

\subsection{Shannon Wavelets in Fourier Domain}

In order to define the multiscale analysis, based on Shannon wavelets, we need to define the Hilbert space of functions that can be reconstructed by them. The Shannon scaling function owns a very simple expression in the Fourier domain, therefore it would be easier to define the scalar product in Fourier domain. To this purpose we define the Fourier transform of the function $f(x) \in L_{2}(\mathbb{R})$, and its inverse transform as

$$
\widehat{f}(\omega)=\widehat{f(x)} \stackrel{\text { def }}{=} \frac{1}{2 \pi} \int_{-\infty}^{\infty} f(x) e^{-i \omega x} d x, \quad f(x)=\int_{-\infty}^{\infty} \widehat{f}(\omega) e^{i \omega x} d \omega .
$$

The Fourier transform of (4.1) give us [23]

$$
\left\{\begin{array}{l}
\widehat{\varphi}(\omega)=\frac{1}{2 \pi} \chi(\omega+3 \pi)=\left\{\begin{array}{cc}
1 /(2 \pi), & -\pi \leq \omega<\pi \\
0, & \text { elsewhere }
\end{array}\right. \\
\widehat{\psi}(\omega)=\frac{1}{2 \pi} e^{i \omega / 2}[\chi(2 \omega)+\chi(-2 \omega)]
\end{array}\right.
$$

with

$$
\chi(\omega)=\left\{\begin{array}{lc}
1, & 2 \pi \leq \omega<4 \pi \\
0, & \text { elsewhere }
\end{array}\right.
$$

The Fourier transform fulfills many interesting properties and among them the following:

$$
\widehat{f(a x)}=\frac{1}{a} \widehat{f}\left(\frac{\omega}{a}\right), \quad \widehat{f(x-b)}=e^{-i b \omega} \widehat{f}(\omega), \quad \widehat{\frac{d^{n}}{d x^{n}} f(x)}=(i \omega)^{n} \widehat{f}(\omega) .
$$

So that for the dilated and translated instances of scaling/wavelet function, in the frequency domain, are

$$
\left\{\begin{array}{l}
\widehat{\varphi}_{k}^{n}(\omega)=\frac{2^{-n / 2}}{2 \pi} e^{i \omega k / 2^{n}} \chi\left(\omega / 2^{n}+3 \pi\right) \\
\widehat{\psi}_{k}^{n}(\omega)=\frac{2^{-n / 2}}{2 \pi} e^{i \omega(k+1 / 2) / 2^{n}}\left[\chi\left(\omega / 2^{n-1}\right)+\chi\left(-\omega / 2^{n-1}\right)\right] .
\end{array}\right.
$$

For the integer order derivatives of scaling and wavelet, according to (4.17), it is

$$
\frac{d^{\ell}}{d x^{\ell}} \varphi_{k}^{n}(x)=(i \omega)^{\ell} \widehat{\varphi}_{k}^{n}(\omega), \quad \widehat{\frac{d^{\ell}}{d x^{\ell}} \psi_{k}^{n}}(x)=(i \omega)^{\ell} \widehat{\psi}_{k}^{n}(\omega)
$$

and, thanks to (4.18), we get

$$
\left\{\begin{aligned}
\widehat{\frac{d^{\ell}}{d x^{\ell}} \varphi_{k}^{n}}(x)= & (i \omega)^{\ell} \frac{2^{-n / 2}}{2 \pi} e^{i \omega k / 2^{n}} \chi\left(\omega / 2^{n}+3 \pi\right), \\
\widehat{\frac{d^{\ell}}{d x^{\ell}} \psi_{k}^{n}(x)=} & (i \omega)^{\ell} \frac{2^{-n / 2}}{2 \pi} e^{i \omega(k+1 / 2) / 2^{n}}\left[\chi\left(\omega / 2^{n-1}\right)\right. \\
& \left.+\chi\left(-\omega / 2^{n-1}\right)\right] .
\end{aligned}\right.
$$


The simple form of these derivative will help us to easily define also the fractional derivatives of these functions. Moreover, as we will see in the next section they form a basis for the $L_{2}(\mathbb{R})$ functions.

\subsection{Wavelet Analysis and Synthesis}

Both families of Shannon scaling and wavelet are $L_{2}(\mathbb{R})$ functions, therefore for each $f(x) \in L_{2}(\mathbb{R})$ and $g(x) \in L_{2}(\mathbb{R})$, the inner product is defined as

$$
\langle f, g\rangle \stackrel{\text { def }}{=} \int_{-\infty}^{\infty} f(x) \overline{g(x)} d x
$$

where the bar stands for the complex conjugate. By taking into account the Parseval theorem

$$
\int_{-\infty}^{\infty} f(x) \overline{g(x)} d x=2 \pi \int_{-\infty}^{\infty} \widehat{f}(\omega) \overline{\widehat{g}(\omega)} d \omega
$$

it is

$$
\langle f, g\rangle \stackrel{\text { def }}{=} \int_{-\infty}^{\infty} f(x) \overline{g(x)} d x=2 \pi \int_{-\infty}^{\infty} \widehat{f}(\omega) \overline{\widehat{g}(\omega)} d \omega=2 \pi\langle\widehat{f}, \widehat{g}\rangle,
$$

Shannon wavelets fulfill the following orthogonality properties (for the proof see e.g., $[23,24]$ )

$$
\begin{aligned}
& \left\langle\psi_{k}^{n}(x), \psi_{h}^{m}(x)\right\rangle=\delta^{n m} \delta_{h k},\left\langle\varphi_{k}^{0}(x), \varphi_{h}^{0}(x)\right\rangle=\delta_{k h}, \\
& \left\langle\varphi_{k}^{0}(x), \psi_{h}^{m}(x)\right\rangle=0, \quad m \geq 0,
\end{aligned}
$$

$\delta^{n m}, \delta_{h k}$ being the Kroenecker symbols.

Let $\mathcal{B} \subset L_{2}(\mathbb{R})$ the set of functions $f(x)$ in $L_{2}(\mathbb{R})$ such that the integrals

$$
\left\{\begin{array}{l}
\alpha_{k} \stackrel{\text { def }}{=}\left\langle f(x), \varphi_{k}(x)\right\rangle \stackrel{(4.22)}{=} \int_{-\infty}^{\infty} f(x) \overline{\varphi_{k}^{0}(x)} d x \\
\beta_{k}^{n} \stackrel{\text { def }}{=}\left\langle f(x), \psi_{k}^{n}(x)\right\rangle \stackrel{(4.22)}{=} \int_{-\infty}^{\infty} f(x) \overline{\psi_{k}^{n}(x)} d x,
\end{array}\right.
$$

exist with finite values, then it can be shown [23, 24, 27, 44], that the series

$$
f(x)=\sum_{h=-\infty}^{\infty} \alpha_{h} \varphi_{h}(x)+\sum_{n=0}^{\infty} \sum_{k=-\infty}^{\infty} \beta_{k}^{n} \psi_{k}^{n}(x)
$$

converges to $f(x)$. So that each function $f(x) \in \mathcal{B} \subset L_{2}(\mathbb{R})$ can be expressed as the wavelet series (4.25), and it is fully characterized by the wavelet coefficient $\alpha_{h}, \beta_{k}^{n}$.

According to (4.22) the coefficients can be also computed in the Fourier domain [24] so that, together with (4.24) we can alternatively use the integrals

$$
\left\{\begin{aligned}
\alpha_{k}= & \int_{-\pi}^{\pi} \widehat{f}(\omega) e^{i \omega k} d \omega, \\
\beta_{k}^{n}= & 2^{-n / 2}\left[\int_{2^{n} \pi}^{2^{n+1} \pi} \widehat{f}(\omega) e^{i \omega(k+1 / 2) / 2^{n}} d \omega\right. \\
& \left.+\int_{-2^{n+1} \pi}^{-2^{n} \pi} \widehat{f}(\omega) e^{i \omega(k+1 / 2) / 2^{n}} d \omega\right] .
\end{aligned}\right.
$$

In the frequency domain, Equation (4.25) gives [24]

$$
\begin{aligned}
\widehat{f}(\omega) & =\frac{1}{2 \pi} \chi(\omega+3 \pi) \sum_{h=-\infty}^{\infty} \alpha_{h} e^{i \omega h} \\
& +\frac{1}{2 \pi} \chi\left(\omega / 2^{n-1}\right) \sum_{n=0}^{\infty} \sum_{k=-\infty}^{\infty} 2^{-n / 2} \beta_{k}^{n} e^{i \omega(k+1 / 2) / 2^{n}} \\
& +\frac{1}{2 \pi} \chi\left(-\omega / 2^{n-1}\right) \sum_{n=0}^{\infty} \sum_{k=-\infty}^{\infty} 2^{-n / 2} \beta_{k}^{n} e^{i \omega(k+1 / 2) / 2^{n}}
\end{aligned}
$$

When the upper bound for the series of (4.25), is finite, then we have the approximation

$$
f(x) \cong \sum_{h=-K}^{K} \alpha_{h} \varphi_{h}(x)+\sum_{n=0}^{N} \sum_{k=-S}^{S} \beta_{k}^{n} \psi_{k}^{n}(x)
$$

The error of the approximation has been estimated in Cattani $[24,26]$.

\section{CONNECTION COEFFICIENTS AND DERIVATIVES}

Let us assume that a function $f(x) \in \mathcal{B}$, so that $f(x)$ is a function belonging to the Hilbert space based on Shannon wavelets and thus being represented in the form of (4.25). In this section we will give the explicit form of the $n$-order integer derivative $f^{(n)}(x)$ and the sinc fractional order derivative $D_{S}^{\alpha} f(x)$. In order to get these derivatives we need to compute the $\ell$-th integer order derivatives of the Shannon family (scaling and wavelet functions) $\varphi_{h}(x), \psi_{k}^{n}(x)$ and the sinc-fractional derivative. The Equations (4.20) already give us the expression of the $\ell$-order derivative in the Fourier domain. In the following sections we will give the explict form of these derivatives also in the space domain.

\subsection{Integer Order Derivatives of the Shannon Wavelets}

It can be shown that the integer order derivatives of the Shannon family can be expressed as orthogonal wavelet series $[23,24,26]$ as follows: 
Definition 6. The integer $n$-order derivative of the Shannon scaling and wavelet functions are

$$
\left\{\begin{array}{l}
\frac{d^{\ell}}{d x^{\ell}} \varphi_{h}(x)=\sum_{k=-\infty}^{\infty} \lambda_{h k}^{(\ell)} \varphi_{k}(x) \\
\frac{d^{\ell}}{d x^{\ell}} \psi_{h}^{m}(x)=\sum_{n=0}^{\infty} \sum_{k=-\infty}^{\infty} \gamma_{h k}^{(\ell) m n} \psi_{k}^{n}(x)
\end{array}\right.
$$

being

$$
\lambda_{k h}^{(\ell)} \equiv\left\langle\frac{d^{\ell}}{d x^{\ell}} \varphi_{k}^{0}(x), \varphi_{h}^{0}(x)\right\rangle, \quad \gamma_{k h}^{(\ell) n m} \equiv\left\langle\frac{d^{\ell}}{d x^{\ell}} \psi_{k}^{n}(x), \psi_{h}^{m}(x)\right\rangle,
$$

the connection coefficients [21, 23, 45-50].

It should be noticed that the connection coefficients are not symmetric. In fact it is

$$
\left\langle\frac{d^{\ell}}{d x^{\ell}} \varphi_{k}^{0}(x), \varphi_{h}^{0}(x)\right\rangle=\frac{d^{\ell}}{d x^{\ell}}\left\langle\varphi_{k}^{0}(x), \varphi_{h}^{0}(x)\right\rangle-\left\langle\varphi_{k}^{0}(x), \frac{d^{\ell}}{d x^{\ell}} \varphi_{h}^{0}(x)\right\rangle,
$$

and by taking into account (4.23), there follows that

$$
\lambda_{k h}^{(\ell)}=-\lambda_{h k}^{(\ell)} \quad h \neq k
$$

Analogously we have for the coefficients

$$
\gamma_{k h}^{(\ell) n m}=-\gamma_{h k}^{(\ell) n m}
$$

The connection coefficients can be easily computed so that it can be shown $[21,23,24]$

Theorem 1. The connection coefficients $(5.2)_{1}$ of the Shannon scaling functions $\varphi_{k}(x)$ are

$\lambda_{k h}^{(\ell)}= \begin{cases}(-1)^{k-h+\ell} \frac{i^{\ell}}{2 \pi} \sum_{s=1}^{\ell} \frac{\ell ! \pi^{s}}{s ![i(k-h)]^{\ell-s+1}}\left[(-1)^{s}-1\right], & k \neq h \\ \frac{i^{\ell} \pi^{\ell+1}}{2 \pi(\ell+1)}\left[1+(-1)^{\ell}\right], & k=h,\end{cases}$

when $\ell \geq 1$. When $\ell=0$, it is

$$
\lambda_{k h}^{(0)}=\delta_{k h}
$$

For the proof see e.g., [23].

Analogously, by defining the sign-function $\mu(x)=\operatorname{sign}(x)$, it can be shown that

Theorem 2. The connection coefficients $(5.2)_{2}$ of the Shannon wavelets $\psi_{k}^{n}(x)$ are for $\ell \geq 1$, and

$$
\gamma_{k h}^{(0) n m}=\delta_{k h} \delta^{n m}
$$

$\ell=0$ respectively.

For the proof see [23].

As a consequence of Equations (5.3),(5.8) the $\ell$-order derivative of the basic functions (4.11) are

$$
\left\{\begin{aligned}
\frac{d^{\ell}}{d x^{\ell}} \varphi(x) & =\sum_{k=-\infty}^{\infty} \lambda_{0 k}^{(\ell)} \varphi_{k}(x), \\
\frac{d^{\ell}}{d x^{\ell}} \psi(x) & =\sum_{n=0}^{\infty} \sum_{k=-\infty}^{\infty} \gamma_{0 h}^{(\ell) 0 n} \psi_{h}^{n}(x) \\
& =\sum_{h=-\infty}^{\infty} \gamma_{0 h}^{(\ell) 00} \psi_{h}^{0}(x),
\end{aligned}\right.
$$

with

$$
\lambda_{0 k}^{(\ell)}= \begin{cases}(-1)^{k+\ell} \frac{i^{\ell}}{2 \pi} \sum_{s=1}^{\ell} \frac{\ell ! \pi^{s}}{s !(i k)^{\ell-s+1}}\left[(-1)^{s}-1\right] & , k \neq 0 \\ \frac{i^{\ell} \pi^{\ell+1}}{2 \pi(\ell+1)}\left[1+(-1)^{\ell}\right] & , k=0,\end{cases}
$$

and

$$
\left\{\begin{aligned}
\gamma_{0 h}^{(\ell) 00}= & \mu(h)\left\{\sum_{s=1}^{\ell+1}(-1)^{[1+\mu(h)](2 \ell-s+1) / 2}\right. \\
& \times \frac{\ell ! \ell^{\ell-s} \pi^{\ell-s}}{(\ell-s+1) !|h|^{s}}(-1)^{-s-2 h} 2^{-s-1} \times\left\{2 ^ { \ell + 1 } \left[(-1)^{4 h+s}\right.\right. \\
& \left.\left.\left.+(-1)^{\ell}\right]-2^{s}\left[(-1)^{h+\ell}+(-1)^{3 h+s}\right]\right\}\right\}, h \neq 0 \\
\gamma_{0 h}^{(\ell) 00}= & {\left[i^{\ell} \frac{\pi^{\ell} 2^{-1}}{\ell+1}\left(2^{\ell+1}-1\right)\left(1+(-1)^{\ell}\right)\right], h=0 }
\end{aligned}\right.
$$

In particular it is

$$
\lambda^{(\ell)} \stackrel{\text { def }}{=} \lambda_{00}^{(\ell)}=\frac{i^{\ell} \pi^{\ell+1}}{2 \pi(\ell+1)}\left[1+(-1)^{\ell}\right]
$$

It can be easily shown that $\lambda^{(\ell)}=0$ for odd $\ell$ so that we have

$$
\lambda^{(\ell)}=\left\{\begin{array}{ll}
(-1)^{s} \frac{\pi^{2 s}}{2 s+1} & , \ell=2 s \\
0 & , \ell=2 s+1
\end{array} \quad(s=1,2, \ldots)\right.
$$


For instance according to (5.6), (5.7) a good approximation of the 2nd order derivative of $\varphi(x)$ is

$$
\begin{aligned}
\frac{d^{2}}{d x^{2}} \varphi(x) \cong & \sum_{k=-2}^{2} \lambda_{0 k}^{(\ell)} \varphi_{k}(x)=-\frac{1}{2} \varphi_{-2}(x)+2 \varphi_{-1}(x)-\frac{1}{3} \pi^{2} \varphi(x) \\
& +2 \varphi_{1}(x)-\frac{1}{2} \varphi_{2}(x) .
\end{aligned}
$$

Also for higher derivatives with high amplitude, the approximation is quite good. For instance according to (5.6), (5.7) for the 7-th derivative of $\varphi(x)$ a quite good approximation is obtained with 15 terms

$$
\frac{d^{\ell}}{d x^{\ell}} \varphi(x) \cong \sum_{k=-7}^{7} \lambda_{0 k}^{(\ell)} \varphi_{k}(x)
$$

\subsection{Properties of Connection Coefficients}

The connection coefficients own many interesting properties like e.g., the following for the scaling functions

Theorem 3. The connection coefficients (5.3) are defined recursively by

$$
\lambda_{k h}^{(\ell+1)}= \begin{cases}\frac{\ell+1}{k-h} \lambda_{k h}^{(\ell)}-(-1)^{k-h} \frac{i^{\ell} \pi^{\ell+1}}{k-h}\left[(-1)^{\ell}+1\right], & k \neq h \\ i \pi \frac{\ell+1}{\ell+2} \lambda_{k h}^{(\ell)}+\frac{(-i)^{\ell+1} \pi^{\ell+1}}{\ell+2}, & k=h,\end{cases}
$$

Proof: see [26].

Analogously for the coefficients $\gamma$.

Theorem 4. The connection coefficients (5.8) are recursively given by the matrix at the lowest scale level:

$$
\gamma_{k h}^{(\ell) n n}=2^{\ell(n-1)} \gamma_{k h}^{(\ell) 11} .
$$

Proof: see [26].

Moreover we can easily check that

$$
\gamma_{k h}^{(2 \ell+1) n n}=-\gamma_{h k}^{(2 \ell+1) n n}, \quad \gamma_{k h}^{(2 \ell) n n}=\gamma_{h k}^{(2 \ell) n n} .
$$

\subsection{Taylor Series}

By using the connection coefficients, and taking into account that the basic functions, according to (5.1), are $\mathcal{C}^{\infty}$-functions, it is easy to show the following theorem:

Theorem 5. Let $f(x) \in \mathcal{B} \subset L_{2}(\mathbb{R})$ the $\ell \geq 1$ order derivative is given by

$$
f^{(\ell)}(x)=\sum_{h, k=-\infty}^{\infty} \alpha_{h} \lambda_{h k}^{(\ell)} \varphi_{k}(x)+\sum_{n, m=0}^{\infty} \sum_{k, s=-\infty}^{\infty} \beta_{k}^{n} \gamma_{s k}^{(\ell) m n} \psi_{s}^{m}(x)
$$

where the coefficients $\alpha_{h}, \beta_{k}^{n}$ are given by (4.24) (or (4.26)) and the connection coefficients are given by (5.3), (5.8).
Proof: The proof easily follows from Equations (4.25), (5.1).

Theorem 6. If $f(x) \in B_{\psi} \subset L_{2}(\mathbb{R})$ and $f(x) \in \mathcal{C}^{S}$ the Taylor series of $f(x)$ in $x_{0}$ is

$$
\begin{aligned}
f(x) & =f\left(x_{0}\right)+\sum_{r=1}^{\infty}\left[\sum_{h, k=-\infty}^{\infty} \alpha_{h} \lambda_{h k}^{(r)} \varphi_{k}\left(x_{0}\right)\right. \\
& \left.+\sum_{n=0}^{\infty} \sum_{k, s=-\infty}^{\infty} 2^{r(n-1)} \beta_{k}^{n} \gamma_{s k}^{(r) 11} \psi_{s}^{n}\left(x_{0}\right)\right] \frac{\left(x-x_{0}\right)^{r}}{r !}
\end{aligned}
$$

being $\alpha_{h}$ and $\beta_{k}^{n}$ given by (4.24), (4.26).

Proof: From (4.25), the $\ell$-order derivative $(\ell \leq S)$ is

$$
\begin{aligned}
f^{(\ell)}(x)= & \sum_{h=-\infty}^{\infty} \alpha_{h} \frac{d^{\ell}}{d x^{\ell}} \varphi_{h}(x)+\sum_{n=0}^{\infty} \sum_{k=-\infty}^{\infty} \beta_{k}^{n} \frac{d^{\ell}}{d x^{\ell}} \psi_{k}^{n}(x), \\
\stackrel{(5.1)}{=} & \sum_{h=-\infty}^{\infty} \alpha_{h} \sum_{k=-\infty}^{\infty} \lambda_{h k}^{(\ell)} \varphi_{k}(x)+\sum_{n=0}^{\infty} \sum_{k=-\infty}^{\infty} \beta_{k}^{n} \sum_{m=-\infty}^{\infty} \\
& \sum_{s=-\infty}^{\infty} \gamma^{(\ell) m n}{ }_{s k}^{(\ell)} \psi_{s}^{m}(x), \\
= & \sum_{h, k=-\infty}^{\infty} \alpha_{h} \lambda_{h k}^{(\ell)} \varphi_{k}(x)+\sum_{n, m=0}^{\infty} \sum_{k, s=-\infty}^{\infty} \beta_{k}^{n} \gamma_{s k}^{(\ell) m n} \psi_{s}^{m}(x),
\end{aligned}
$$

so that by taking into account (5.12) the proof follows.

By a suitable choice of the initial point $x_{0}$ Equation (5.14) can be simplified. For instance, at the integers, $x_{0}=j,(j \in \mathbb{Z})$, according to Equations (4.12), (5.12) it is

$$
\begin{aligned}
f(x) \cong f(j)+\sum_{r=1}^{S} & {\left[\sum_{h=-\infty}^{\infty} \alpha_{h} \lambda_{h j}^{(r)}+\sum_{n=0}^{\infty}\right.} \\
& \left.\sum_{k, s=-\infty}^{\infty} \frac{2^{r(n-1)+1+n / 2}}{\left(2^{n+1} h-2 s-1\right) \pi} \beta_{k}^{n} \gamma_{s k}^{(r) 11} \psi_{s}^{n}(h)\right] \frac{(x-j)^{r}}{r !}
\end{aligned}
$$

In particular, for $x_{0}=j=0$, Equation (5.14) gives

$$
\begin{aligned}
f(x)= & f(0)+\sum_{r=1}^{\infty}\left[\sum_{h, k=-\infty}^{\infty} \alpha_{h} \lambda_{h k}^{(r)} \varphi_{k}(0)+\sum_{n=0}^{\infty}\right. \\
& \left.\sum_{k, s=-\infty}^{\infty} 2^{r(n-1)} \beta_{k}^{n} \gamma_{s k}^{(r) 11} \psi_{s}^{n}(0)\right] \frac{x^{r}}{r !} \\
= & f(0)+\sum_{r=1}^{\infty}\left[\sum_{h=-\infty}^{\infty} \alpha_{h} \lambda_{h 0}^{(r)}+\sum_{n=0}^{\infty}\right. \\
& \left.\sum_{k, s=-\infty}^{\infty} 2^{r(n-1)} \beta_{k}^{n} \gamma_{s k}^{(r) 11} \psi_{s}^{n}(0)\right] \frac{x^{r}}{r !}
\end{aligned}
$$


and since

$$
\psi_{s}^{n}(0)=(-1)^{s} \frac{2^{1+n / 2}}{(-2 s-1) \pi}, \quad(-2 k-1 \neq 0)
$$

we get

$$
\begin{aligned}
f(x)= & f(0)+\sum_{r=1}^{\infty}\left[\sum_{h=-\infty}^{\infty} \alpha_{h} \lambda_{h 0}^{(r)}+\sum_{n=0}^{\infty} \sum_{k, s=-\infty}^{\infty}\right. \\
& \left.\frac{(-1)^{s+1} 2^{n(r+1 / 2)+1-r}}{(2 s+1) \pi} \beta_{k}^{n} \gamma_{s k}^{(r) 11}\right] \frac{x^{r}}{r !}
\end{aligned}
$$

with $\lambda_{h 0}^{(r)}$ given by (5.7) and $\gamma_{s k}^{(r) 11}$ by (5.8) respectively. So that each function $f(x) \in \mathcal{B} \subset L_{2}(\mathbb{R})$, can be easily expressed as a power series, when the finite values of the wavelet coefficients $\alpha_{h}$, $\beta_{k}^{n}$ are given, according to (4.24),(4.26).

There follows, in particular, the Taylor power series for the basic functions $\varphi(x), \psi(x)$ :

$$
\left\{\begin{array}{l}
\varphi(x)=1+\sum_{r=1}^{\infty}\left(\sum_{h=-\infty}^{\infty} \lambda_{h 0}^{(r)}\right) \frac{x^{r}}{r !} \\
\psi(x)=-\frac{2}{\pi}+\sum_{r=1}^{\infty}\left(\sum_{n=0}^{\infty} \sum_{k, s=-\infty}^{\infty} \frac{(-1)^{s+1} 2^{n(r+1 / 2)+1-r}}{(2 s+1) \pi} \delta_{k}^{n} \gamma_{s k}^{(r) 11}\right) \frac{x^{r}}{r !}
\end{array}\right.
$$

being $\psi(0)=-\frac{2}{\pi}$, according to (4.1).

For a fixed $r$ the series

$$
\Lambda^{r} \stackrel{\text { def }}{=} \sum_{h=-\infty}^{\infty} \lambda_{h 0}^{(r)}, \quad r \geq 1, h \neq=0
$$

is converging, as can be easily shown by using Equation (5.7). In particular it is

$$
\begin{aligned}
& \lambda_{h 0}^{(1)}=-\frac{(-1)^{h}}{h}, \quad \lambda_{h 0}^{(2)}=-2 \frac{(-1)^{h}}{h^{2}}, \quad \lambda_{h 0}^{(3)}=(-1)^{h}\left(\frac{\pi^{2}}{h}-\frac{6}{h^{3}}\right), \\
& \lambda_{h 0}^{(4)}=(-1)^{h}\left(\frac{4 \pi^{2}}{h^{2}}-\frac{24}{h^{4}}\right), \quad \lambda_{h 0}^{(5)}=(-1)^{h}\left(\frac{\pi^{4}}{h}-\frac{20 \pi^{2}}{h^{3}}+\frac{120}{h^{5}}\right) \\
& \lambda_{h 0}^{(6)}=-(-1)^{h}\left(\frac{6 \pi^{4}}{h^{2}}-\frac{120 \pi^{2}}{h^{4}}+\frac{720}{h^{6}}\right), \ldots
\end{aligned}
$$

Moreover, since for odd $r$ it is $\Lambda^{(r)}=0$ while for even $r$ it is

$$
\Lambda^{2 r}=2 \sum_{h=0}^{\infty} \lambda_{h 0}^{(r)}, \quad r \geq 1, h \neq=0
$$

so that $\varphi(x)$ can be written as the power series

$$
\varphi(x)=\sum_{r=0}^{\infty} \frac{\Lambda^{r}}{r !} x^{r}, \quad\left(\Lambda^{0} \stackrel{\text { def }}{=} 1\right)
$$

The first (approximated) values of the coefficients $\Lambda$ are:

$$
\Lambda^{0}=1, \Lambda^{1}=0.69, \Lambda^{2}=1.64,, \Lambda^{3}=-1.43,
$$

$$
\Lambda^{4}=-9.74,, \Lambda^{5}=6.19
$$

In particular, the Taylor series for the wavelet function $\psi(x)$ can be also easily computed as follows:

$$
\begin{gathered}
\psi(x)=\quad \psi(0)+\sum_{\ell=1}^{\infty}\left(\frac{d^{\ell} \psi(x)}{d x^{\ell}}\right)_{x=0} \frac{x^{\ell}}{\ell !} \\
\stackrel{(5.1),(5.6)}{=}-\frac{2}{\pi}+\sum_{\ell=1}^{\infty}\left(\sum_{k=-\infty}^{\infty} \gamma_{0 k}^{(\ell) 00} \psi_{k}^{0}(0)\right) \frac{x^{\ell}}{\ell !} \\
\stackrel{(4.13)}{=}-\frac{2}{\pi}+\sum_{\ell=1}^{\infty}\left(\sum_{k=-\infty}^{\infty} \gamma_{0 k}^{(\ell) 00}(-1)^{k+1} \frac{2}{(2 k+1) \pi}\right) \frac{x^{\ell}}{\ell !}
\end{gathered}
$$

that is

$$
\psi(x)=-\frac{2}{\pi}+\sum_{\ell=1}^{\infty}\left(\sum_{k=-\infty}^{\infty}(-1)^{k+1} \frac{2}{(2 k+1) \pi} \gamma_{0 k}^{(\ell) 00}\right) \frac{x^{\ell}}{\ell !}
$$

\section{SINC-FRACTIONAL DERIVATIVES FOR THE FUNCTIONS $F(X) \in \mathcal{B} \subset L_{2}(\mathbb{R})$}

The sinc fractional derivative (3.10) is defined by a sinc kernel over an infinite domain. Although the sinc-function is the basic function for Shannon wavelet, this kernel is not a Shannon scaling function for the reason that the sinc function depends on the fractional (non-integer) order of derivative. On the other hand as shown by the Equation (5.13) the $n$-integer order derivative can be written as a linear combination of $\varphi_{k}(x), \psi_{k}^{m}(x)$. Therefore, in order to give an explicit form to (3.10) as a function of Shannon wavelet and connection coefficients, we need to compute the scalar products of Shannon scaling and wavelet with sinc-function.

\subsection{Scalar Products of the Shannon Scaling and Wavelet Functions With Sinc-Function}

In this section we consider the scalar product of the sinc function with the Shannon scaling and wavelet functions and corresponding derivatives. We need these products to compute the sinc fractional derivatives.

\subsubsection{Scalar Product of the Shannon Scaling Function With Sinc-Function}

Let us assume $a, b \in \mathbb{R}$ and show the following theorem:

Theorem 7. The scalar product ot the scaling functions $\varphi_{k}(\tau)$ with the sinc-function is

$$
\left\langle\operatorname{sinc}(a \tau-b), \varphi_{k}(\tau)\right\rangle= \begin{cases}\frac{2 \pi}{a} \operatorname{sinc}(b+k), & a \geq 1 \\ \frac{2 \pi}{a^{2}} \operatorname{sinc} \frac{(b+k)}{a}, & a<1 .\end{cases}
$$


Proof: It is by definition

$$
\left\langle\operatorname{sinc}(a \tau-b), \varphi_{k}(\tau)\right\rangle=\int_{-\infty}^{\infty} \operatorname{sinc}(a \tau-b) \varphi_{k}(\tau) d \tau .
$$

According to (4.22) this product can be easily done in the Fourier domain,

$$
\left.\left\langle\operatorname{sinc}(a \tau-b), \varphi_{k}(\tau)\right\rangle=2 \pi\langle\operatorname{sinc} \widehat{(a \tau}-b), \widehat{\varphi_{k}(\tau)}\right\rangle
$$

from where by using the properties (4.17) it is

$$
\operatorname{sinc} \widehat{(a \tau-b)}=\frac{1}{a} \widehat{\operatorname{sinc}}\left(\frac{\omega}{a}\right) e^{-i b \omega}, \quad \widehat{\operatorname{sinc}}\left(\frac{\omega}{a}\right) \stackrel{(4.16)}{=} \frac{1}{2 \pi} \chi\left(\frac{\omega}{a}+3 \pi\right)
$$

so that by taking into account $(4.18)_{1}$

$\left\langle\operatorname{sinc}(a \tau-b), \varphi_{k}(\tau)\right\rangle=2 \pi \frac{1}{a} \frac{1}{2 \pi}\left\langle e^{-i b \omega} \chi\left(\frac{\omega}{a}+3 \pi\right), e^{-i k \omega} \chi(\omega+3 \pi)\right\rangle$.

The integral can be easily computed, being

$$
\begin{aligned}
& \left\langle e^{-i b \omega} \chi\left(\frac{\omega}{a}+3 \pi\right), e^{-i k \omega} \chi(\omega+3 \pi)\right\rangle \\
= & \left.\int_{-\infty}^{\infty} e^{-i(b+k) \omega} \chi\left(\frac{\omega}{a}+3 \pi\right) \chi(\omega+3 \pi) d \omega\right\rangle .
\end{aligned}
$$

There follows that, if $\frac{1}{a} \leq 1$ it is

$$
\begin{gathered}
\int_{-\infty}^{\infty} e^{-i(b+k) \omega} \chi\left(\frac{\omega}{a}+3 \pi\right) \chi(\omega+3 \pi) d \omega=\int_{-\infty}^{\infty} e^{-i(b+k) \omega} \chi(\omega+3 \pi) d \omega \\
=\int_{-\pi}^{\pi} e^{-i(b+k) \omega} d \omega=2 \pi \operatorname{sinc}(b+k)
\end{gathered}
$$

While for $\frac{1}{a}>1$ it is

$$
\begin{gathered}
\int_{-\infty}^{\infty} e^{-i(b+k) \omega} \chi\left(\frac{\omega}{a}+3 \pi\right) \chi(\omega+3 \pi) d \omega=\int_{-\infty}^{\infty} e^{-i(b+k) \omega} \chi\left(\frac{\omega}{a}+3 \pi\right) d \omega \\
=\int_{-\frac{\pi}{a}}^{\frac{\pi}{a}} e^{-i(b+k) \omega} d \omega=\frac{2 \pi}{a} \operatorname{sinc} \frac{(b+k)}{a} .
\end{gathered}
$$

From where there easily follows the result (6.1).

In particular, according to (4.17), it is

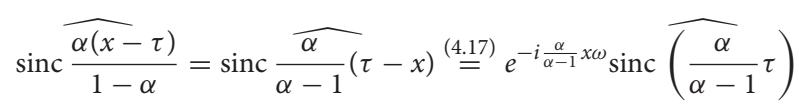

that is

$$
\operatorname{sinc} \frac{\widehat{\alpha(x}-\tau)}{1-\alpha}=\frac{\alpha-1}{\alpha} e^{-i \frac{\alpha}{\alpha-1} x \omega} \widehat{\operatorname{sinc}}\left(\frac{\alpha-1}{\alpha} \omega\right) .
$$

Since we have

$$
\widehat{\operatorname{sinc}(\tau)} \stackrel{(4.16)}{=} \frac{1}{2 \pi} \chi(\omega+3 \pi)
$$

there follows

$$
\operatorname{sinc} \frac{\widehat{\alpha(x-\tau)}}{1-\alpha}=\frac{1}{2 \pi} \frac{\alpha-1}{\alpha} e^{-i \frac{\alpha}{\alpha-1} x \omega} \chi\left(\frac{\alpha-1}{\alpha} \omega+3 \pi\right)
$$

so that, by taking

$$
a=\frac{\alpha}{\alpha-1}, \quad b=\frac{\alpha}{\alpha-1} x
$$

from (6.1) we get

$$
\begin{aligned}
& \left\langle\operatorname{sinc}\left(\frac{\alpha}{\alpha-1} \tau-\frac{\alpha}{\alpha-1} x\right), \varphi_{k}(\tau)\right\rangle \\
= & \begin{cases}\frac{2 \pi(\alpha-1)}{\alpha} \operatorname{sinc}\left(\frac{\alpha}{\alpha-1} x+k\right), & \alpha \geq 1 \\
\frac{2 \pi(\alpha-1)^{2}}{\alpha^{2}} \operatorname{sinc}\left(x+k \frac{\alpha-1}{\alpha}\right), & \alpha<1 .\end{cases}
\end{aligned}
$$

Analogously we can give an explicit form to the scalar product of the integer $n$-order derivative.

Theorem 8. The scalar product of the $n$-th order derivative $\varphi_{k}^{(n)}(\tau)$ with the sinc-function is

$$
\left\langle\operatorname{sinc}(a \tau-b), \varphi_{k}^{(n)}(\tau)\right\rangle= \begin{cases}\int_{-\pi}^{\pi}(i \omega)^{n} e^{-i(b+k) \omega} d \omega, & a \geq 1 \\ \int_{-\frac{\pi}{a}}^{\frac{\pi}{a}}(i \omega)^{n} e^{-i(b+k) \omega} d \omega, & a<1\end{cases}
$$

Proof: It is

$$
\left\langle\operatorname{sinc}(a \tau-b), \varphi_{k}^{(n)}(\tau)\right\rangle=\int_{-\infty}^{\infty} \operatorname{sinc}(a \tau-b) \varphi_{k}^{(n)}(\tau) d \tau .
$$

According to (4.22) this product can be easily done in the Fourier domain,

$$
\left\langle\operatorname{sinc}(a \tau-b), \varphi_{k}^{(n)}(\tau)\right\rangle=2 \pi\left\langle\operatorname{sinc} \widehat{(a \tau-b)}, \widehat{\varphi_{k}^{(n)}(\tau)}\right\rangle
$$

from where by using the properties (4.17) it is

$$
\begin{aligned}
\operatorname{sinc}(\widehat{a \tau}-b) & =\frac{1}{2 \pi a} e^{-i b \omega} \chi\left(\frac{\omega}{a}+3 \pi\right) \\
\widehat{\varphi_{k}^{(n)}(\tau)} & =(i \omega)^{n} \widehat{\varphi_{k}(\tau)}=(i \omega)^{n} e^{-i k \omega} \chi(\omega+3 \pi)
\end{aligned}
$$

so that by taking into account $(4.18)_{1}$

$$
\begin{aligned}
& \left\langle\operatorname{sinc}(a \tau-b), \varphi_{k}^{(n)}(\tau)\right\rangle \\
= & 2 \pi \frac{1}{a} \frac{1}{2 \pi}\left\langle e^{-i b \omega} \chi\left(\frac{\omega}{a}+3 \pi\right),(i \omega)^{n} e^{-i k \omega} \chi(\omega+3 \pi)\right\rangle .
\end{aligned}
$$

The integral can be easily computed, being

$$
\left\langle e^{-i b \omega} \chi\left(\frac{\omega}{a}+3 \pi\right), e^{-i k \omega} \chi(\omega+3 \pi)\right\rangle
$$




$$
\left.=\int_{-\infty}^{\infty}(i \omega)^{n} e^{-i(b+k) \omega} \chi\left(\frac{\omega}{a}+3 \pi\right) \chi(\omega+3 \pi) d \omega\right\rangle .
$$

There follows that, if $\frac{1}{a} \leq 1$ it is

$$
\begin{gathered}
\int_{-\infty}^{\infty}(i \omega)^{n} e^{-i(b+k) \omega} \chi\left(\frac{\omega}{a}+3 \pi\right) \chi(\omega+3 \pi) d \omega \\
=\int_{-\infty}^{\infty}(i \omega)^{n} e^{-i(b+k) \omega} \chi(\omega+3 \pi) d \omega \\
=\int_{-\pi}^{\pi}(i \omega)^{n} e^{-i(b+k) \omega} d \omega .
\end{gathered}
$$

While for $\frac{1}{a}>1$ it is

$$
\begin{gathered}
\int_{-\infty}^{\infty}(i \omega)^{n} e^{-i(b+k) \omega} \times\left(\frac{\omega}{a}+3 \pi\right) \chi(\omega+3 \pi) d \omega \\
=\int_{-\infty}^{\infty}(i \omega)^{n} e^{-i(b+k) \omega} \chi\left(\frac{\omega}{a}+3 \pi\right) d \omega \\
=\int_{-\frac{\pi}{a}}^{\frac{\pi}{a}}(i \omega)^{n} e^{-i(b+k) \omega} d \omega .
\end{gathered}
$$

From where there easily follows the result (6.1).

In particular, for the first derivative it is

$$
\int_{-\pi}^{\pi}(i \omega) e^{-i(b+k) \omega} d \omega=\frac{2 \pi}{b+k}[\operatorname{sinc}(b+k)-\cos (b+k) \pi]
$$

and

$\int_{-\frac{\pi}{a}}^{\frac{\pi}{a}}(i \omega)^{n} e^{-i(b+k) \omega} d \omega=\frac{2 \pi}{a(b+k)}\left[\operatorname{sinc} \frac{b+k}{a}-a \cos \frac{b+k}{a} \pi\right]$

so that

$$
\begin{aligned}
\left\langle\operatorname{sinc}(a \tau-b), \varphi_{k}^{\prime}(\tau)\right\rangle \\
\begin{cases}\frac{2 \pi}{b+k}[\operatorname{sinc}(b+k)-\cos (b+k) \pi], & a \geq 1 \\
\frac{2 \pi}{a(b+k)}\left[\operatorname{sinc} \frac{b+k}{a}-a \cos \frac{b+k}{a} \pi\right], & a<1 .\end{cases}
\end{aligned}
$$

In general the scalar product of the $n$-order derivative (with $n>$ 1 ) is given by the lengthly computation of the integrals (6.3). In the next section we will see that this computation can be avoided by using the connection coefficients.

\subsubsection{Scalar Product of the Shannon Wavelets With Sinc Function}

Analogously, for the derivative of the wavelet function it can be easily shown that
Theorem 9. Let $a, b \in \mathbb{R}$, the scalar product ot the wavelet functions $\psi_{k}^{n}(\tau)$ with the sinc-function is

$$
\begin{gathered}
\left\langle\operatorname{sinc}(a \tau-b), \psi_{k}^{n}(\tau)\right\rangle=\Gamma_{k}^{n}(\tau, a, b) \stackrel{\text { def }}{=} \frac{2^{n / 2+1}}{a \pi\left(2^{n+1} b-2 k-1\right)} \times \\
\times \begin{cases}0 & , a<1 \\
\sin \left(\frac{1}{2} a\left(2 b-2^{-n}(1+2 k)\right)\right) \pi+\cos \left(-2^{n} b+k\right) \pi & , 2^{n}<a<2^{n+1} \\
\sin \left(-2^{n+1} b+2 k\right) \pi+\cos \left(-2^{n} b+k\right) \pi & , 2^{n+1} \geq a .\end{cases}
\end{gathered}
$$

Proof: It is

$$
\left\langle\operatorname{sinc}(a \tau-b), \psi_{k}^{n}(\tau)\right\rangle=\int_{-\infty}^{\infty} \operatorname{sinc}(a \tau-b) \psi_{k}^{n}(\tau) d \tau .
$$

According to (4.22) this product can be easily done in the Fourier domain,

$$
\left.\left\langle\operatorname{sinc}(a \tau-b), \psi_{k}^{n}(\tau)\right\rangle=2 \pi\langle\operatorname{sinc} \widehat{(a \tau}-b), \widehat{\psi_{k}^{n}}\right\rangle
$$

from where by using the properties (4.17), it is

$$
\operatorname{sinc}(a \tau-b)=\frac{1}{a} \frac{1}{2 \pi} \times\left(\frac{\omega}{a}+3 \pi\right) e^{-i b \omega}
$$

so that by taking into account $(4.18)_{2}$

$$
\begin{array}{r}
\left\langle\operatorname{sinc}(a \tau-b), \psi_{k}^{n}(\tau)\right\rangle=\frac{2^{-n / 2}}{2 a \pi}\left\langle e^{-i b \omega} \chi\left(\frac{\omega}{a}+3 \pi\right), e^{i \omega(k+1 / 2) / 2^{n}}\right. \\
\left.\left[\chi\left(\omega / 2^{n-1}\right)+\chi\left(-\omega / 2^{n-1}\right)\right]\right\rangle
\end{array}
$$

that is

$$
\begin{array}{r}
\left\langle\operatorname{sinc}(a \tau-b), \psi_{k}^{n}(\tau)\right\rangle=\frac{2^{-n / 2}}{2 a \pi}\left\langle e^{i \omega\left(k+1 / 2-2^{n} b\right) / 2^{n}} \chi\left(\frac{\omega}{a}+3 \pi\right),\right. \\
\left.\left[\chi\left(\omega / 2^{n-1}\right)+\chi\left(-\omega / 2^{n-1}\right)\right]\right\rangle .
\end{array}
$$

Let us notice that the value of the scalar product (and then of the integral) depends on the non-vanishing values of the characteristic function. On the other hands the characteristic function $\chi$ depends on the values $a, n, k$. In fact the nonvanishing values of the characteristic functions are

$$
\begin{aligned}
& \chi\left(\frac{\omega}{a}+3 \pi\right)=1, \text { if }-a \pi<\omega<a \pi \\
& \chi\left(\omega / 2^{n-1}\right)=1, \quad \text { if } 2^{n} \pi<\omega<2^{n}(2 \pi) \\
& \chi\left(-\omega / 2^{n-1}\right)=1, \text { if }-2^{n}(2 \pi)<\omega<-2^{n} \pi .
\end{aligned}
$$

There follow three cases:

1. $a \pi<\pi$. In this case $a<1$, the characteristic functions have some disjoint intervals and the scalar product vanishes

$$
\left\langle e^{i \omega\left(k+1 / 2-2^{n} b\right) / 2^{n}} \chi\left(\frac{\omega}{a}+3 \pi\right),\left[\chi\left(\omega / 2^{n-1}\right)+\chi\left(-\omega / 2^{n-1}\right)\right]\right\rangle=0 .
$$


2. $2^{n} \pi<a \pi<2^{n}(2 \pi)$. Here we have $2^{n}<a<2^{n+1}$ the integral becomes

$$
\begin{gathered}
\left\langle e^{i \omega\left(k+1 / 2-2^{n} b\right) / 2^{n}} \chi\left(\frac{\omega}{a}+3 \pi\right),\left[\chi\left(\omega / 2^{n-1}\right)+\chi\left(-\omega / 2^{n-1}\right)\right]\right\rangle= \\
=\int_{-a \pi}^{-2^{n} \pi} e^{i \omega\left(k+1 / 2-2^{n} b\right) / 2^{n}} d \omega+\int_{2^{n} \pi}^{a \pi} e^{i \omega\left(k+1 / 2-2^{n} b\right) / 2^{n}} d \omega \\
=\frac{2^{n+2}}{2^{n+1} b-2 k-1}\left[\sin \left(\frac{1}{2} a\left(2 b-2^{-n}(1+2 k)\right)\right) \pi+\cos \left(-2^{n} b+k\right) \pi\right] .
\end{gathered}
$$

3. $2^{n}(2 \pi) \leq a \pi$. We have $2^{n+1} \leq a$ so that the integral is

$$
\begin{aligned}
& \left\langle e^{i \omega\left(k+1 / 2-2^{n} b\right) / 2^{n}} \chi\left(\frac{\omega}{a}+3 \pi\right),\left[\chi\left(\omega / 2^{n-1}\right)+\chi\left(-\omega / 2^{n-1}\right)\right]\right\rangle= \\
& =\int_{-2^{n+1} \pi}^{-2^{n} \pi} e^{i \omega\left(k+1 / 2-2^{n} b\right) / 2^{n}} d \omega+\int_{2^{n} \pi}^{2^{n+1} \pi} e^{i \omega\left(k+1 / 2-2^{n} b\right) / 2^{n}} d \omega \\
& =\frac{2^{n+2}}{2^{n+1} b-2 k-1}\left[\sin \left(-2^{n+1} b+2 k\right) \pi+\cos \left(-2^{n} b+k\right) \pi\right] .
\end{aligned}
$$

From where we obtain (6.5).

\subsection{Sinc-Fractional Derivative of Functions $f(x) \in \mathcal{B} \subset L_{2}(\mathbb{R})$}

In order to define the sinc-fractional derivative for the functions $f(x) \in \mathcal{B}$, according to the reconstruction formula (4.25) we need to compute the sinc-fractional derivative of the scaling and wavelet functions. These derivatives are given by the following theorems.

Theorem 10. The sinc-fractional derivative (3.10) of the scaling function $\varphi_{k}(x)$ is

$$
\begin{aligned}
D_{S}^{\alpha} \varphi_{h}(x)= & -2 \pi P(\alpha) \sum_{k=-\infty}^{\infty} \lambda_{h k}^{(n)} \times \\
& \begin{cases}\operatorname{sinc}\left(\frac{\alpha}{\alpha-1} x+k\right), & \alpha \geq 1 \\
\frac{\alpha-1}{\alpha} \operatorname{sinc}\left(x+\frac{\alpha}{1-\alpha} k\right), & \alpha<1\end{cases}
\end{aligned}
$$

Proof: Starting from the definition (3.10) of the sinc-derivative it is

$$
D_{S}^{\alpha} \varphi_{h}(x) \stackrel{(3.10)}{=} \frac{\alpha P(\alpha)}{1-\alpha} \int_{-\infty}^{\infty} \operatorname{sinc} \frac{\alpha(x-\tau)}{1-\alpha} \frac{d^{n}}{d \tau^{n}} \varphi_{h}(\tau) d \tau,
$$

According to (4.22), the derivatives (6.7), can be written also as scalar product,

$$
D_{S}^{\alpha} \varphi_{h}(x)=\frac{\alpha P(\alpha)}{1-\alpha}\left\langle\operatorname{sinc} \frac{\alpha(x-\tau)}{1-\alpha}, \frac{d^{n}}{d \tau^{n}} \varphi_{h}(\tau)\right\rangle,
$$

From here by using the integer order derivatives (5.1) we

$$
D_{S}^{\alpha} \varphi_{h}(x)=\frac{\alpha P(\alpha)}{1-\alpha} \sum_{k=-\infty}^{\infty} \lambda_{h k}^{(n)}\left\langle\operatorname{sinc} \frac{\alpha(x-\tau)}{1-\alpha}, \varphi_{k}(\tau)\right\rangle
$$

So that the computation of the sinc-fractional derivative of a function, that can be expressed as wavelet series, is reduced to the computation of the scalar product:

$$
\left\langle\operatorname{sinc} \frac{\alpha(x-\tau)}{1-\alpha}, \varphi_{k}(\tau)\right\rangle, \quad 0 \leq n-1<\alpha<n
$$

which is given by (6.1) with

$$
a=\frac{\alpha}{\alpha-1}, \quad b=\frac{\alpha}{\alpha-1} x .
$$

It can be easily seen that these inequalities imply

$$
a \geq 1 \Longrightarrow \alpha \geq 1, \quad a<1 \Longrightarrow \alpha<1 \text {. }
$$

From these inequalities, by taking into account $(6.1),(6.9)$, there easily follows (6.6).

Analogously, we have for the Shannon wavelet fractional derivatives the following

Theorem 11. The sinc-fractional derivative (3.10) of the Shannon wavelets $\psi_{h}^{m}(x)$ is

$$
\begin{gathered}
D_{S}^{\alpha} \psi_{h}^{m}(x)=\frac{\alpha P(\alpha)}{1-\alpha} \sum_{s=0}^{\infty} \\
\sum_{k=-\infty}^{\infty} \gamma_{h k}^{(n) m s} \frac{2^{s / 2+1}(\alpha-1)}{\alpha \pi\left(2^{s+1} \frac{\alpha}{\alpha-1}-2 k-1\right)} \times
\end{gathered}
$$

$$
\times \begin{cases}0, & a<1 \\ \sin \left(\frac{1}{2} \frac{\alpha}{\alpha-1}\left(2 \frac{\alpha}{\alpha-1} x-2^{-s}(1+2 k)\right)\right) \pi & \\ +\cos \left(-2^{s} \frac{\alpha}{\alpha-1} x+k\right) \pi, & 2^{s}<a<2^{s+1} \\ \sin \left(-2^{s+1} b+2 k\right) \pi+\cos \left(-2^{s} b+k\right) \pi, & 2^{s+1} \geq a\end{cases}
$$

Proof: From the definition (3.10) it is

$$
D_{S}^{\alpha} \psi_{h}^{m}(x) \stackrel{(3.10)}{=} \frac{\alpha P(\alpha)}{1-\alpha} \int_{-\infty}^{\infty} \operatorname{sinc} \frac{\alpha(x-\tau)}{1-\alpha} \frac{d^{n}}{d \tau^{n}} \psi_{h}^{m}(\tau) d \tau
$$

According to (4.22), this derivative can be written also as scalar product,

$$
D_{S}^{\alpha} \psi_{h}^{m}(x)=\frac{\alpha P(\alpha)}{1-\alpha}\left\langle\operatorname{sinc} \frac{\alpha(x-\tau)}{1-\alpha}, \frac{d^{n}}{d \tau^{n}} \psi_{h}^{m}(\tau)\right\rangle,
$$

so that by taking into account (5.1) which gives the integer order derivatives it is

$$
D_{S}^{\alpha} \psi_{h}^{m}(x)=\frac{\alpha P(\alpha)}{1-\alpha} \sum_{s=0}^{\infty} \sum_{k=-\infty}^{\infty} \gamma_{h k}^{(n) m s}\left\langle\operatorname{sinc} \frac{\alpha(x-\tau)}{1-\alpha}, \psi_{k}^{s}(\tau)\right\rangle
$$

and using (6.5) we get (6.11). 
Equations (6.6) and (6.11) enable us to compute explicitly the sinc-fractional derivative of any function belonging to the Hilbert space $\mathcal{B} \subset L_{2}(\mathbb{R})$. In fact, let $f(x) \in \mathcal{B}$ a function such that it can be represented as the wavelet series (4.25). Its sinc-fractional derivative can be computed according to

Theorem 12. The sinc-fractional derivative of the wavelet representation (4.25) of function $f(x) \in \mathcal{B} \subset L_{2}(\mathbb{R})$, is given by

$$
\begin{gathered}
D_{S}^{v} f(x)=-2 \pi P(v) \sum_{h=-\infty}^{\infty} \alpha_{h} \sum_{k=-\infty}^{\infty} \lambda_{h k}^{(n)} \\
\times\left\{\begin{array}{l}
\sin c\left(\frac{v}{v-1} x+k\right), \quad v \geq 1 \\
\frac{v-1}{v} \operatorname{sinc}\left(x+\frac{v}{1-v} k\right), \quad v<1 \\
+\frac{v P(v)}{1-v} \sum_{m=0}^{\infty} \sum_{k=-\infty}^{\infty} \beta_{h}^{m} \sum_{s=0}^{\infty} \\
\sum_{k=-\infty}^{\infty} \gamma^{(n) m s} \frac{2^{s / 2+1}(v-1)}{v \pi\left(2^{s+1} \frac{v}{v-1}-2 k-1\right)} \times \\
\sin \left(\frac{1}{2} \frac{v}{v-1}\left(2 \frac{v}{v-1} x-2^{-s}(1+2 k)\right) \pi\right. \\
+\cos \left(-2^{s} \frac{v}{v-1} x+k\right) \pi, \\
\sin \left(-2^{s+1} \frac{v}{v-1} x\right. \\
+2 k) \pi+\cos \left(-2^{s} \frac{v}{v-1} x+k\right) \pi,
\end{array} \quad 2^{s}<v<2^{s+1}\right. \\
0
\end{gathered}
$$

with $0 \leq n-1<v<n$.

Proof : Let us start from Equation (3.10), and the representation (4.25), because of the linearity of the operator we have

$$
D_{S}^{v} f(x)=\sum_{h=-\infty}^{\infty} \alpha_{h} D_{S}^{v} \varphi_{h}(x)+\sum_{m=0}^{\infty} \sum_{k=-\infty}^{\infty} \beta_{h}^{m} D_{S}^{v} \psi_{h}^{m}(x)
$$

where the wavelet coefficients $\alpha_{h}, \beta_{h}^{m}$ are given by (4.24) [or (4.26)]. From here, by using (6.6) and (6.11), we get (6.15).

In particular, with $n=1$ we have

Theorem 13. The sinc-fractional derivative of the wavelet representation (4.25) of function $f(x) \in \mathcal{B} \subset L_{2}(\mathbb{R})$, with order $0<v<1$, is

$$
\begin{array}{r}
D_{S}^{v} f(x)=2 \pi P(v) \frac{1-v}{v} \sum_{h=-\infty}^{\infty} \alpha_{h} \sum_{k=-\infty}^{\infty} \lambda_{h k}^{(1)} \operatorname{sinc}\left(x+\frac{v}{1-v} k\right), \\
0<v<1
\end{array}
$$

Proof: Follows directly from Equation (6.15).

\subsection{Example: Fractional Derivative of the Gaussian Function}

In order to show the efficiency of the proposed method for the computation of a fractional derivative, let us consider the function $e^{-x^{2}}$. A good approximation of this function, in terms of Shannon wavelet expansion (4.25), can be obtained as

$$
\begin{aligned}
e^{-x^{2}} \cong \sum_{h=-1}^{1} \alpha_{h} \varphi_{h}(x)+\sum_{n=0}^{0} \sum_{h=-1}^{1} \beta_{h}^{n} \psi_{h}^{n}(x) \\
\cong \alpha_{-1} \varphi_{-1}(x)+\alpha_{0} \varphi(x)+\alpha_{1} \varphi_{1}(x)+ \\
+\beta_{-1}^{0} \psi_{-1}^{0}(x)+\beta_{0}^{0} \psi_{0}^{0}(x)+\beta_{1}^{0} \psi_{1}^{0}(x)
\end{aligned}
$$

where

$\alpha_{-1}=\alpha_{1}=0.123, \alpha_{0}=0.30, \psi_{-1}^{0}=\psi_{1}^{0}=0.004, \psi_{0}^{0}=0.001$.

If we neglect also the detailed coefficients $\beta_{k}^{n}$ the approximate Shannon wavelet representation is

$$
e^{-x^{2}} \cong 0.123 \varphi_{-1}(x)+0.30 \varphi(x)+0.123 \varphi_{1}(x) .
$$

From (6.16) we have

$$
\begin{array}{r}
D_{S}^{v} e^{-x^{2}} \cong 2 \pi P(v) \frac{1-v}{v} \sum_{h=-1}^{1} \alpha_{h} \sum_{k=-1}^{1} \lambda_{h k}^{(1)} \operatorname{sinc}\left(x+\frac{v}{1-v} k\right), \\
0<v<1 .
\end{array}
$$

The matrix $\lambda_{h k}^{(1)}$, according to (5.3) is

$$
\begin{aligned}
\lambda_{-1-1}^{(1)}=\lambda_{00}^{(1)}=\lambda_{11}^{(1)}=0, \lambda_{0-1}^{(1)}=-\lambda_{-10}^{(1)}=\lambda_{10}^{(1)} & =-\lambda_{01}^{(1)}=1, \\
\lambda_{-11}^{(1)} & =-\lambda_{1-1}^{(1)}=\frac{1}{2}
\end{aligned}
$$

so that by simplifying we get

$$
\begin{array}{r}
D_{S}^{v} e^{-x^{2}} \cong 2 \pi P(v) \frac{1-v}{v}\left(\alpha_{0}-\frac{1}{2} \alpha_{1}\right)\left[\operatorname{sinc}\left(x-\frac{v}{1-v}\right)\right. \\
\left.-\operatorname{sinc}\left(x+\frac{v}{1-v}\right)\right], 0<v<1
\end{array}
$$

that is

$$
\begin{array}{r}
D_{S}^{v} e^{-x^{2}} \cong 0.47 \pi P(v) \frac{1-v}{v}\left[\operatorname{sinc}\left(x-\frac{v}{1-v}\right)\right. \\
\left.-\operatorname{sinc}\left(x+\frac{v}{1-v}\right)\right], 0<v<1 .
\end{array}
$$

\section{CONCLUSION}

Sinc function is playing a fundamental role in mathematics and physics. Due to the many properties of this function it deserves a 
special role in applications. In recent years some Authors have proposed [20] a fractional derivative based on this function. Moreover a wavelet theory based on the sinc function has been settled thus extending the many features of the Sinc. In this paper the sinc-fractional derivative has been extended to the Shannon wavelet space, in order to give the explicit analytical form of the fractional derivatives of functions belonging to the wavelet space. It has been shown that the sinc-fractional derivative is the most

\section{REFERENCES}

1. Dalir M, Bashour M. Applications of fractional calculus. Appl Math Sci. (2010) 4:1021-32.

2. Tarasov VE. Fractional vector calculus and fractional Maxwells equations. Ann Phys. (2008) 323:2756-78. doi: 10.1016/j.aop.2008.04.005

3. Tarasov VE. Electromagnetic fields on fractals. Mod Phys Lett A (2006) 21:1587-600. doi: 10.1142/S0217732306020974

4. Liu K, Hu R-J, Cattani C, Xie GN, Yang X-J, Zhao Y. Local fractional Z transforms with applications to signals on Cantor sets. Abstr Appl Anal. (2014) 2014:638648. doi: 10.1155/2014/638648

5. Yang X-J, Srivastava HM, He J-H, Baleanu D. Cantor-type cylindricalcoordinate method for differential equations with local fractional derivatives. Phys Lett A (2013) 377:1696-700. doi: 10.1016/j.physleta.2013.04.012

6. Yang AM, Yang X-J, Li ZB. Local fractional series expansion method for solving wave and diffusion equations on Cantor sets, Abstr Appl Anal. (2013) 2013:351057. doi: 10.1155/2013/351057

7. Zhao Y, Baleanu D, Cattani C, Cheng DF, Yang X-J. Maxwells equations on Cantor sets: a local fractional approach. Adv High Energy Phys. (2013) 2013:686371. doi: 10.1155/2013/686371

8. Baleanu D, Inc M, Yusuf A, Aliyu AI. Space-time fractional Rosenou-Haynam equation: lie symmetry analysis, explicit solutions and conservation laws. $A d v$ Diff Equat. (2018) 2018:46. doi: 10.1186/s13662-018-1468-3

9. Baleanu D, Inc M, Yusuf A, Aliyu AI. Lie symmetry analysis, exact solutions and conservation laws for the time fractional Caudrey Dodd Gibbon Sawada Kotera equation. Commun Nonlinear Sci Numer Simul. (2018) 59:222-34. doi: 10.1016/j.cnsns.2017.11.015

10. Baleanu D, Inc M, Yusuf A, Aliyu AI. Lie symmetry analysis and conservation laws for the time fractional simplified modified Kawahara equation. Open Phys. (2018) 16:302-10. doi: 10.1515/phys-2018-0042

11. Baleanu D, Inc M, Yusuf A, Aliyu AI. Optimal system, nonlinear selfadjointness and conservation laws for generalized shallow water wave equation. Open Phys. (2018) 16:364-70. doi: 10.1515/phys-2018-0049

12. Baleanu D, Inc M, Yusuf A, Aliyu AI. Lie symmetry analysis and explicit solutions for the time fractional generalized Burgers-Huxley equation. Opt Quant Electron. (2018) 50:94. doi: 10.1007/s11082-018-1373-8

13. Baleanu D, Inc M, Yusuf A, Aliyu AI. Lie symmetry analysis, exact solutions and conservation laws for the time fractional modified Zakharov Kuznetsov equation. Nonlinear Anal Model Control (2018) 22:861-76. doi: 10.15388/NA.2017.6.9

14. Inc M, Yusuf A, Aliyu AI, Baleanu D. Investigation of the logarithmicKdV equation involving Mittag-Leffler type kernel with Atangana Baleanu derivative. Physica A (2018) 506:520-31. doi: 10.1016/j.physa.2018.04.092

15. Tchier F, Inc M, Yusuf A, Aliyu AI, Baleanu D. Time fractional thirdorder variant Boussinesq system: symmetry analysis, explicit solutions, conservation laws and numerical approximations. Eur Phys J Plus (2018) 133:240. doi: 10.1140/epjp/i2018-12068-0

16. Wang X. Fractional geometric calculus: toward a unified mathematical language for physics and engineering. In: Proceedings of The Fifth Symposium on Fractional Differentiation and its Applications (FDA 12), Hohai University, Nanjing (2012).

17. Li C, Dao X, Guo P. Fractional derivatives in complex planes. Nonlinear Anal Theor Methods Appl. (2009) 71:1857-69. doi: 10.1016/j.na.2009. 01.021 natural and suitable choice of fractional operator when dealing with functions that can be represented as Shannon wavelet series.

\section{AUTHOR CONTRIBUTIONS}

The author confirms being the sole contributor of this work and has approved it for publication.

18. Ortigueira MD. A coherent approach to non-integer order derivatives. Signal Process. ( 2006) 86:2505-15. doi: 10.1016/j.sigpro.2006.02.002

19. Cattani C, Guariglia E. Fractional derivative of the Hurwitz $\zeta$ function and chaotic decay to zero. J King Saud Univ. (2016) 28:75-81. doi: 10.1016/j.jksus.2015.04.003

20. Yang X-J, Gao F, Terneiro Machado JA, Baleanu D. A new fractional derivative involving the normalized sinc function without singular kernel. arXiv:1701.05590 (2017)p. 1-11

21. Cattani C. Connection coefficients of shannon wavelets. Math Model Anal. (2006) 11:1-16. doi: 10.1080/13926292.2006.9637307

22. Cattani C. Shannon Wavelet Analysis. In: Proceedings of the International Conference on Computational Science,ICCS 2007; Lecture Notes in Computer Science, LNCS 4488, Part II. Shi Y, Albada GD van Dongarra J, Sloot PMA, Editors. Berlin; Heidelberg: Springer-Verlag (2007) p. 982-9.

23. Cattani C. Shannon wavelets theory. Math Probl Eng. (2008) 2008:1-24. doi: $10.1155 / 2008 / 164808$

24. Cattani C. Shannon wavelets for the solution of integrodifferential equations. Math Probl Eng. (2010) 2010:1-24. doi: 10.1155/2010/408418

25. Cattani C. Fractional calculus and Shannon Wavelet. Math Probl Eng. (2012) 2012:502812. doi: 10.1155/2012/502812

26. Cattani C. Local Fractional Calculus on Shannon Wavelet Basis. In Fractional Dynamics. Cattani C, Srivastava H, Yang XJ, Editors. Krakow: De Gruyter (2015).

27. Daubechies I. Ten Lectures on Wavelets. Philadelphia, PA: CBMS-NSF Regional Conference Series in Applied Mathematics, SIAM (1992).

28. Podlubny I. Fractional Differential Equations: An Introduction to Fractional Derivatives, Fractional Differential Equations, to Methods of Their Solution and Some of Their Applications. New York, NY: Academic Press (1998).

29. Diethelm K. The Analysis of Fractional Differential Equations. Berlin: Springer (2010).

30. Odibat Z, Shawagfeh NT. Generalized taylors formula. Appl Math Comput. (2007) 186:286-93. doi: 10.1016/j.amc.2006.07.102

31. Bagley R. On the equivalence of the Riemann-Liouville and the Caputo fractional order derivatives in modeling of linear viscoelastic materials. Fract Calculus Appl Anal. (2010) 10:123-6. Available online at: http://hdl.handle. net/10525/1310

32. Baleanu D, Diethelm K, Scalas E, Trujillo JJ. Fractional Calculus: Models and Numerical Methods (Series on Complexity, Nonlinearity and Chaos). Singapore: World Scientific (2012).

33. Baleanu D, Terneiro Machado A. Fractional Dynamics and Control. New York, NY: Springer (2014).

34. Caputo M, Fabrizio M. A new definition of fractional derivative without singular kernel. Prog Fract Diff Appl. (2015) 1:73-85. doi: 10.12785/pfda/ 010201

35. Cattani C, Srivastava H, Yang X-J. Fractional Dynamics, Warsaw; Berlin: De Gruyter Open (2015).

36. de Oliveira EC, Tenreiro Machado JA. A review of definitions for fractional derivatives and integrals. Math Probl Eng. (2014) 2014:238459. doi: $10.1155 / 2014 / 238459$

37. Boyadjiev L, Ishteva M, Scherer R. On the caputo operator of fractional calculus and C-Laguerre function. Math Sci Res J. (2005) 9:161-70.

38. Kamata M, Nakamula A. Riemann-Liouville integrals of fractional order and extended KP hierarchy. J Phys A Math Gen. (2002) 35:9657-70. doi: 10.1088/0305-4470/35/45/312 
39. Ortigueira MD, Terneiro Machado A. What is a fractional derivative. $J$ Comput Phys. (2015) 293:4-13. doi: 10.1016/j.jcp.2014.07.019

40. Ortigueira MD, Terneiro Machado A. Which derivative? Fract Fract. (2017) 1:1-13. doi: 10.3390/fractalfract 1010003

41. Yang X-J, Baleanu D, Srivastava HM. Local Fractional Integral Transforms and Their Applications. New York, NY: Academic Press (2015).

42. Caputo M. Linear model of dissipation whose Q is almost frequency independent-II. Geophys J R Astr Soc. (1967) 13:529-39. doi: 10.1111/j.1365-246X.1967.tb02303.x

43. Atangana A, Baleanu D. New fractional derivative with nonlocal and nonsingular kernel: theory and applications to heat transfer model. Thermal Sci. (2016) 20:763-9. doi: 10.2298/TSCI160111018A

44. Cattani C, Rushchitsky JJ. Wavelet and Wave Analysis as applied to Materials with Micro or Nanostructure. Series on Advances in Mathematics for Applied Sciences. Singapore: World Scientific (2007).

45. Cattani C. Harmonic wavelet solutions of the Schrödinger equation. Int J Fluid Mech Res. (2003) 30:463-72. doi: 10.1615/InterJFluidMechRes.v30. i5.10

46. Cattani C. Harmonic wavelets towards solution of nonlinear PDE. Comput Math Appl. 50:1191-210. doi: 10.1016/j.camwa.2005. 07.001

47. Latto A, Resnikoff HL, Tenenbaum E. The evaluation of connection coefficients of compactly supported wavelets. In: Proc. of the French-USA
Workshop on Wavelets and Turbulence Maday Y, Editor. Springer-Verlag (1992) p. 76-89.

48. Lin EB Zhou X. Connection coefficients on an interval and wavelet solutions of Burgers equation. J Comput Appl Math. (2001) 135:63-78. doi: 10.1016/S0377-0427(00)00562-8

49. Restrepo J, Leaf GK. Wavelet-Galerkin discretization of hyperbolic equations. J Comput Phys. (1995) 122:118-28.

50. Romine CH,Peyton BW. Computing Connection Coefficients of Compactly Supported Wavelets on Bounded Intervals. Computer Science and Mathematics Division; Mathematical Sciences Section; Oak Ridge National Laboratory; Oak Ridge, TN. Technical Report ORNL/TM-13413 (1997) p. 23. Available online at: http://www.ornl.gov/

Conflict of Interest Statement: The author declares that the research was conducted in the absence of any commercial or financial relationships that could be construed as a potential conflict of interest.

Copyright $(2018$ Cattani. This is an open-access article distributed under the terms of the Creative Commons Attribution License (CC BY). The use, distribution or reproduction in other forums is permitted, provided the original author(s) and the copyright owner(s) are credited and that the original publication in this journal is cited, in accordance with accepted academic practice. No use, distribution or reproduction is permitted which does not comply with these terms. 